\title{
Biomass and RRR-a-tocopherol production in Stichococcus bacillaris strain siva2011 in a balloon bioreactor
}

\author{
Ganapathy Sivakumar ${ }^{*}$, Kwangkook Jeong $^{2}$ and Jackson O Lay $\mathrm{Jr}^{3}$
}

\begin{abstract}
Background: Green microalgae represent a renewable natural source of vitamin E. Its most bioactive form is the naturally occurring RRR-a-tocopherol which is biosynthesized in photosynthetic organisms as a single stereoisomer. It is noteworthy that the natural and synthetic a-tocopherols are different biomolecular entities. This article focuses on RRR-a-tocopherol production in Stichococcus bacillaris strain siva2011 biomass in a bioreactor culture with methyl jasmonate (MeJa) elicitor. Additionally, a nonlinear mathematical model was used to quantitatively scale-up and predict the biomass production in a $20 \mathrm{~L}$ balloon bioreactor with dual variables such as time and volume.

Results: Approximately $0.6 \mathrm{mg} / \mathrm{g}$ dry weight (DW) of RRR-a-tocopherol was enhanced in S. bacillaris strain siva2011 biomass with the MeJa $50 \mathrm{\mu L} / \mathrm{L}$ for 24 hrs elicitations when compared to the control. The $R^{2}$ value from the nonlinear model was enhanced up to $95 \%$ when compared to the linear model which significantly improved the accuracy for estimating S. bacillaris strain siva2011 biomass production in a balloon bioreactor.

Conclusions: S. bacillaris strain siva2011 is a new green microalga which biosynthesizes significant amounts of $R R R$-a-tocopherol. Systematically validated dual variable empirical data should provide key insights to multivariable or fourth order modeling for algal biomass scale-up. This bioprocess engineering should provide valuable information for industrial production of RRR-a-tocopherol from green cells.
\end{abstract}

Keywords: Antioxidant, Biomass, Bioreactor, Microalgae, Vitamin E

\section{Background}

$R R R$ - $\alpha$-tocopherol is a lipid soluble small molecule and the biologically active form of natural vitamin E. $R R R-\alpha-$ tocopherol is exclusively biosynthesized by photosynthetic organisms or green cells including algae, plants, and cyanobacteria [1-3]. Plant-based products are a primary source of $R R R$ - $\alpha$-tocopherol in the human diet. For example, hazelnut is one of the richest sources of vitamin E [4]. It is known that vitamin E plays an important role in human nutrition as a natural antioxidant. It was recently proposed that vitamin $\mathrm{E}$ is active against oxidative stress-related diseases [5]. Reportedly, it suppresses telomerase activity in ovarian cancer cells [6]. Vitamin E enhances IL-2 production, gene expression, and is an effective therapeutic adjuvant [7]. Vitamin E

\footnotetext{
* Correspondence: sivakumar@libero.it

${ }^{1}$ Arkansas Biosciences Institute and College of Agriculture and Technology,

Arkansas State University, PO Box 639, Jonesboro, AR 72401, USA

Full list of author information is available at the end of the article
}

deficiency affects both $\mathrm{T}$ and $\mathrm{B}$ immune cell functions [8], the $\alpha$-tocopherol transfer protein ( $\alpha$-TTP) gene, and neurologic dysfunctions. In animal models, vitamin $\mathrm{E}$ mixtures inhibit colon, lung, mammary, and prostate carcinogenesis [9], as well as prevent diabetes [10]. Cosmetic industries also extensively use vitamin $\mathrm{E}$ in skin care products. In addition, $R R R$ - $\alpha$-tocopherol prolongs the shelf life of meat [11]. Thus, the use of $R R R-\alpha$-tocopherol continues to increase in nutraceuticals [12].

Bioreactor technology is the key component for the industrial scale production of bioactive small molecules for pharmaceutical applications. For instance, bioreactor technology was successfully developed and scaled-up to $10,000 \mathrm{~L}$ for commercial-scale production of ginseng roots used for human health-related applications [13,14]. This reactor configuration has also been tested for $R R R-\alpha-$ tocopherol production in lab-scale photosynthetic hazelnut root culture [15]. However, knowledge regarding the bioprocessing of green algal cells for production of $R R R$ - 
$\alpha$-tocopherol in balloon bioreactors is limited. Previously, Stichococcus bacillaris strain siva2011 biomass was scaledup in a lab-scale balloon bioreactor ( $4 \mathrm{~L}-8 \mathrm{~L}$ ), and a linear fitting model for predicting scale-up was proposed [16]. The S. bacillaris strain siva2011 has unique lipid (Figure 1) [16] and vitamin E metabolisms to lead to bioactive $R R R$ - $\alpha$-tocopherol production. A nonlinear model enables more accurate estimates and should provide insight for quantitatively predicating algal biomass accumulation in a large volume bioreactor. The objectives of this study were to: 1$)$ evaluate $R R R$ - $\alpha$-tocopherol content from $S$. bacillaris strain siva2011 biomass with MeJa elicitation; 2) quantitatively predict $S$. bacillaris strain siva2011 DW in a $20 \mathrm{~L}$ bioreactor with simplified stepwise dual variables (time and volume) from $4 \mathrm{~L}$ and $8 \mathrm{~L}$ data using nonlinear regression. This systematic study could provide insight regarding stepwise nonlinear scale-up of $S$. bacillaris strain siva2011 biomass for $R R R$ - $\alpha$-tocopherol production in a balloon bioreactor.

\section{Results and discussions}

Natural vitamin E occurs in two general forms, namely the tocopherols and tocotrienols, which are collectively called tocochromanols. Each has four distinct isoforms having $\alpha, \beta, \gamma$, or $\delta$ substitution on the chromanol. The natural $\alpha$-tocopherol contains three methyl groups on the chromanol moiety at positions 5,7 , and 8 . The phytyl or saturated side chain is attached to the $\mathrm{C}-2$ position of the chromanol ring which has three chiral centers with a single $R R R$ stereoisomer (Figure 2). The chromanol groups have two fused rings, a phenol and a tetrahydropyran, sharing a 2 carbon bridge [17]. These rings are moderately polar, giving them an affinity for the cellular membrane surface while the phytyl tail is hydrophobic and normally associated with membrane lipids [18]. These structural features of $R R R$ - $\alpha$-tocopherol are efficiently acted on by the human hepatic $\alpha$-TTP which is responsible for maintaining plasma $\alpha$-tocopherol concentrations [19].

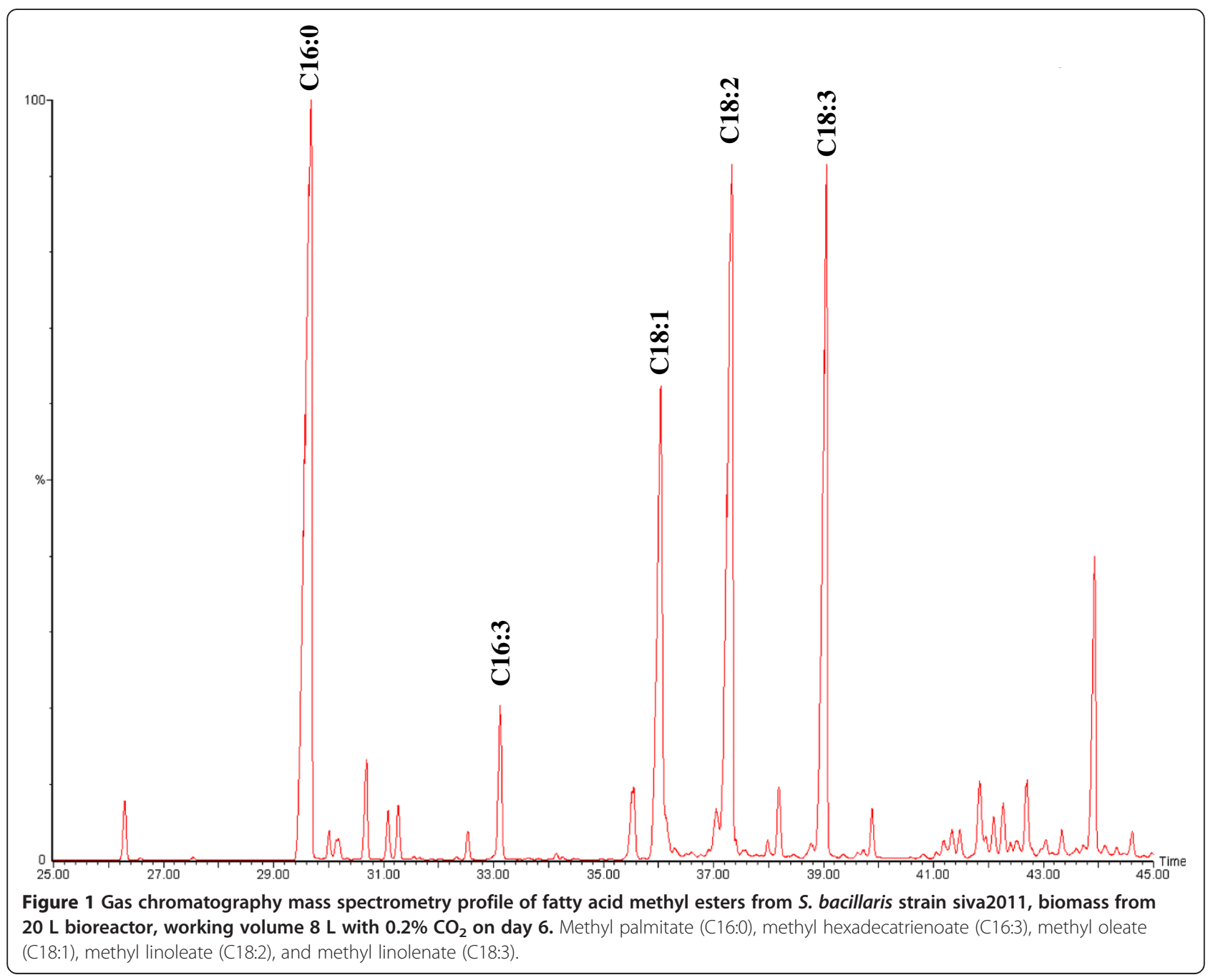




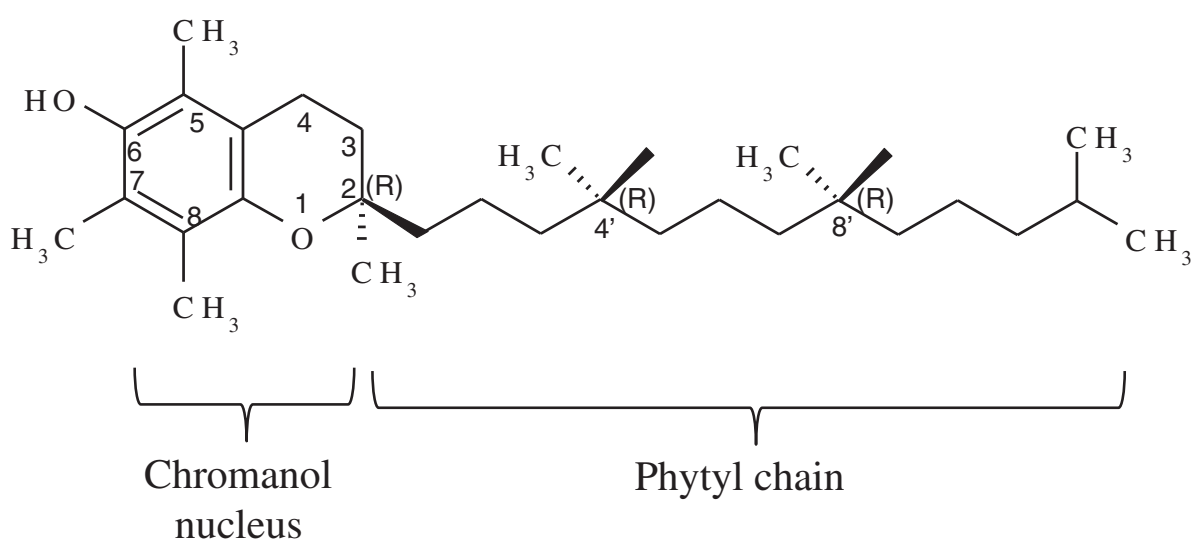

Figure 2 Chemical structure of $R R R$-a-tocopherol.

The synthetic $\alpha$-tocopherol called all-racemic- $\alpha$-tocopherol is not identical to $R R R$ - $\alpha$-tocopherol. It is an equimolar mixture of eight stereoisomers which possess three chiral centers at positions 2', 4', and 8', giving rise to four diastereoisomeric pairs of enantiomers such as $R R R$, RSR, RRS, RSS, SRR, SSR, SRS, and SSS [20]. Moreover, $\alpha$-TTP has a high affinity to $R R R$ - $\alpha$-tocopherol and has a 3-fold greater binding half-life when compared to synthetic $\alpha$-tocopherol [21]. Thus, the bioactivity and the relative safety are different. Human proteins such as enzymes and receptors usually exhibit high stereospecificity [20]. Therefore, the natural $R R R-\alpha$-tocopherol is more bioactive than the synthetic form.

$R R R$ - $\alpha$-tocopherol plays a major role as an antioxidant which prevents lipid peroxidation. In the photosynthetic cells, it may protect photosystem II during photoinhibition and repair chloroplast mechanisms [18]. This is due to the hydroxyl group on the C-6 position which is the active site that donates a hydrogen atom. The phenolic hydrogen atom is capable of scavenging lipid peroxy radicals and quenching singlet oxygen [22]. $R R R$ - $\alpha$-tocopherol is recycled in the photosynthetic cell by cytosolic ascorbate which oxidizes one-electron from the tocopheroxyl radical thus regenerating vitamin E [23]. This mechanism might protect the cell membranes.

$R R R$ - $\alpha$-tocopherol is biosynthesized in photosynthetic cells via two different pathways [24]. The phytyl domain precursor comes from an isoprenoid pathway, and the chromanol domain precursor comes from an alternative shikimate pathway homogentisic acid via complex enzymatic reactions [25]. $R R R-\alpha$-tocopherol is found in the chloroplast envelope, thylakoids, and plastoglobuli of the plastid. The vitamin E biosynthetic pathway has been elucidated in Arabidopsis [26]. The genes associated with vitamin $\mathrm{E}$ biosynthesis in photosynthetic organisms have been well described in literature [3,27]. A significant metabolic engineering effort has been made to improve vitamin $\mathrm{E}$ content both in plants [28] and in cyanobacteria $[29,30]$. Moreover, tocopherol production in plant green callus [31], cell [32], and root cultures [15] have also been reported.

\section{$R R R$-a-tocopherol production in S. bacillaris strain siva2011}

Photosynthetic algae are a potential alternative for production because they biosynthesize an abundance of $R R R$ - $\alpha$-tocopherol. For instance, freshwater Euglena gracilis [33], marine Dunaliella tertiolecta and Tetraselmis suecica [34], model system Chlamydomonas reinhardtii [35], and commercial algae Spirulina platensis [36] have been used to biosynthesize $R R R$ - $\alpha$-tocopherol. The new green alga, S. bacillaris strain siva2011 [16], produces significant amounts of $R R R$ - $\alpha$-tocopherol. This microalga has efficient photosynthetic mechanisms which facilitate the quick biosynthesis of vitamin $\mathrm{E}$.

Tocopherol production can be enhanced by molecular elicitation which is non-transgenic. MeJa is a plant stress volatile signaling molecule which up-regulates several defense-related genes [37]. In plants, jasmonates are biosynthesized via the octadecanoid pathway; exogenous MeJa treatment is up-regulating secondary metabolic pathway genes especially those encoding for stress protection [38]. Therefore, it is used as one of the potential molecular elicitors in plant root culture to enhance several pharmaceutical molecule productions [15]. For instance, MeJa elicitation increases the activity of tyrosine aminotransferase in plant green cell culture which is one of the initial step enzymes involved in tocopherol biosynthesis $[31,39]$. To increase $R R R-\alpha$-tocopherol content in S. bacillaris strain siva2011, MeJa elicitation was also used. The S. bacillaris strain siva2011 characteristics, culture conditions, and bioreactor experimental designs were previously reported for lipid production [16].

Figure 3 illustrates the $R R R$ - $\alpha$-tocopherol content of $S$. bacillaris strain siva2011 biomass under elicitation with various concentrations of MeJa. The unelicited culture 


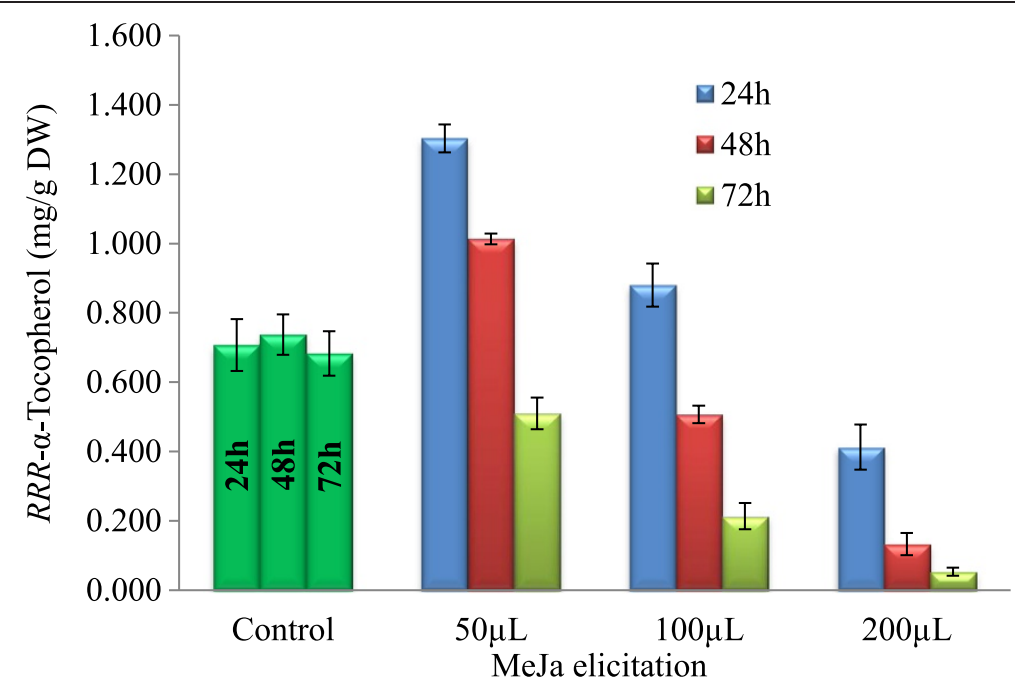

Figure 3 RRR-a-tocopherol content in S. bacillaris strain siva2011, unelicited and methyl jasmonate elicited culture on day 4, 5, and 6.

accumulated $0.7 \mathrm{mg} / \mathrm{g} \mathrm{DW}$ of $R R R$ - $\alpha$-tocopherol which was detected starting during the early exponential growth phase. The lower concentration of MeJa, $50 \mu \mathrm{L} / \mathrm{L}$, after 24 hrs elicitation, enhanced the production of $R R R-\alpha-$ tocopherol to the highest concentration, $1.3 \mathrm{mg} / \mathrm{g} D W$. The higher MeJa concentrations or longer elicitation periods were inhibiting both to the biomass growth and the resultant $R R R$ - $\alpha$-tocopherol production. MeJa can diffuse to cells either by intercellular migration or while in the vapor phase [40]. In plants, MeJa can transport from leaves to roots [41]. The vapor signaling can be transported to distal plants via air, and the intercellular signaling can be transported via vascular process [42]. For instance, in an in vitro root culture the MeJa elicitation could trigger the defensive molecules accumulation via intercellular transport [13-15], whereas in in vivo plants during herbivore attack it can act as a volatile signal [42]. In the algal culture, MeJa elicitation could trigger the $R R R$ - $\alpha$-tocopherol production by either intercellular signaling or both. The optimum concentration can upregulate the tocopherol biosynthetic pathway enzymes in S. bacillaris strain siva2011 which could increase the antioxidant. MeJa elicitation rapidly activates the defensive genes which also down regulates the photosynthetic system genes [38]. In addition, MeJa induces reactive oxygen species (ROS) which alter the mitochondrial and chloroplast dynamics [43]. Thus, the higher MeJa concentrations or longer elicitation periods can produce uncontrolled ROS which can precede chloroplast or photosynthetic dysfunctions which could be inhibiting the tocopherol biosynthesis and biomass accumulation in S. bacillaris strain siva2011. In green cells, chloroplasts are an essential organelle for energy capture and transduction; a decline in photosynthetic activity is closely related to the decrease in the biomass. The typical MeJa elicited cells' symptoms were loss of chlorophyll, which causes the decline in the net photosynthetic rate, and degradation of ribulose bisphosphate carboxylase, etc. [44]. For instance, $100 \mu \mathrm{L}$ MeJa at 9 hrs elicitation altered chloroplast morphology and function which is associated with cell death [43]. Even though $R R R$ - $\alpha$-tocopherol indirectly regulates the amounts of jasmonic acid [18], the decline in chloroplast efficiency could down regulate the $R R R-\alpha$-tocopherol metabolism. This suggests that higher MeJa concentrations or longer elicitation could be cytotoxic beyond what was studied.

\section{Nonlinear regression}

Compared to green adventitious roots, photosynthetic algae have a higher ability to biosynthesize $R R R$ - $\alpha$-tocopherol in bioreactor cultures. The balloon bioreactor is a liquid-phase reactor with enhanced geometry and efficient fluid flow dynamics which could help provide higher mass transfer efficiency [14]. When compared to other photobioreactors, the balloon bioreactor had a larger headspace which efficiently captured light and enhanced photosynthesis [16]. Thus, S. bacillaris strain siva2011 was scaled-up in a balloon bioreactor (Figure 4) to investigate enhanced biomass accumulation. Of the four concentrations of $\mathrm{CO}_{2}$ tested, $0.2 \%$ yielded the highest biomass of $3.45 \mathrm{~g} / \mathrm{L}$ in $4 \mathrm{~L}$ and $3.79 \mathrm{~g} / \mathrm{L}(\mathrm{DW})$ in $8 \mathrm{~L}$ on day 6 [16]. The $R R R$ - $\alpha$-tocopherol production was unchanged by the $0.2 \% \mathrm{CO}_{2}$ (data not shown).

To quantitatively predict larger-scale algal biomass production based on lab-scale test data, the following stepwise structured approach was proposed [45,46]: 1) to set up the simplest model to linearize with dual variables; 2) to model a nonlinear regression with dual variables; and 3) to demonstrate multiple variables based on a nonlinear regression. Although scale-up predication 


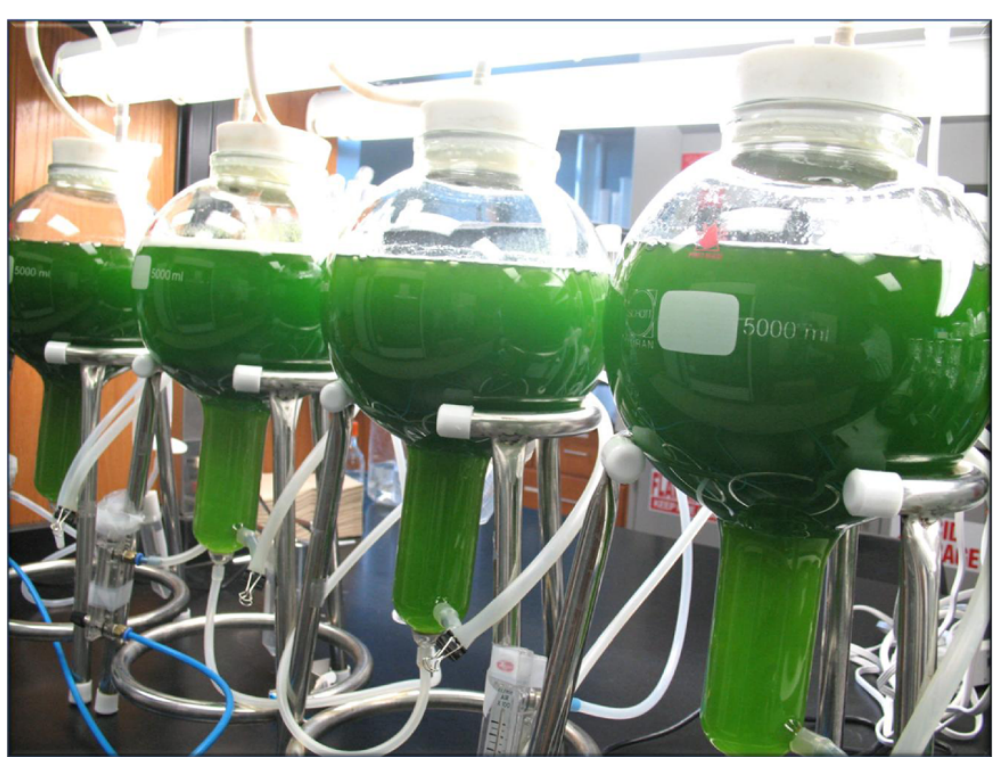

Figure 4 Biomass production of S. bacillaris strain siva2011 in 5 L balloon type bioreactors (working volume 4 L).

requires multiple variables, the 6 days algal culture does not significantly utilize all the media components. Therefore, selection of important dual variables can give insight on the efficiency of parameter selection for lab-scale validation and initial scale-up prediction. In addition, the multiple linear regression models might not incorporate the underlying nonlinear relationships [47]. So, in this study the second systematic approach was conducted to evaluate nonlinear modeling for scale-up prediction in a $20 \mathrm{~L}$ balloon reactor using dual variables. Nonlinear regression models are more important tools than linear models because they provide better parsimony, interpretability, and prediction [48]. Figure 5 illustrates nonlinear modeling of predicted S. bacillaris strain siva2011 biomass accumulation in a $20 \mathrm{~L}$ balloon bioreactor with $0.2 \% \mathrm{CO}_{2}$. This model was generated using $4 \mathrm{~L}$ to $8 \mathrm{~L}$ data of S. bacillaris strain siva2011 and shows only small discrepancies between measured and predicted data. The

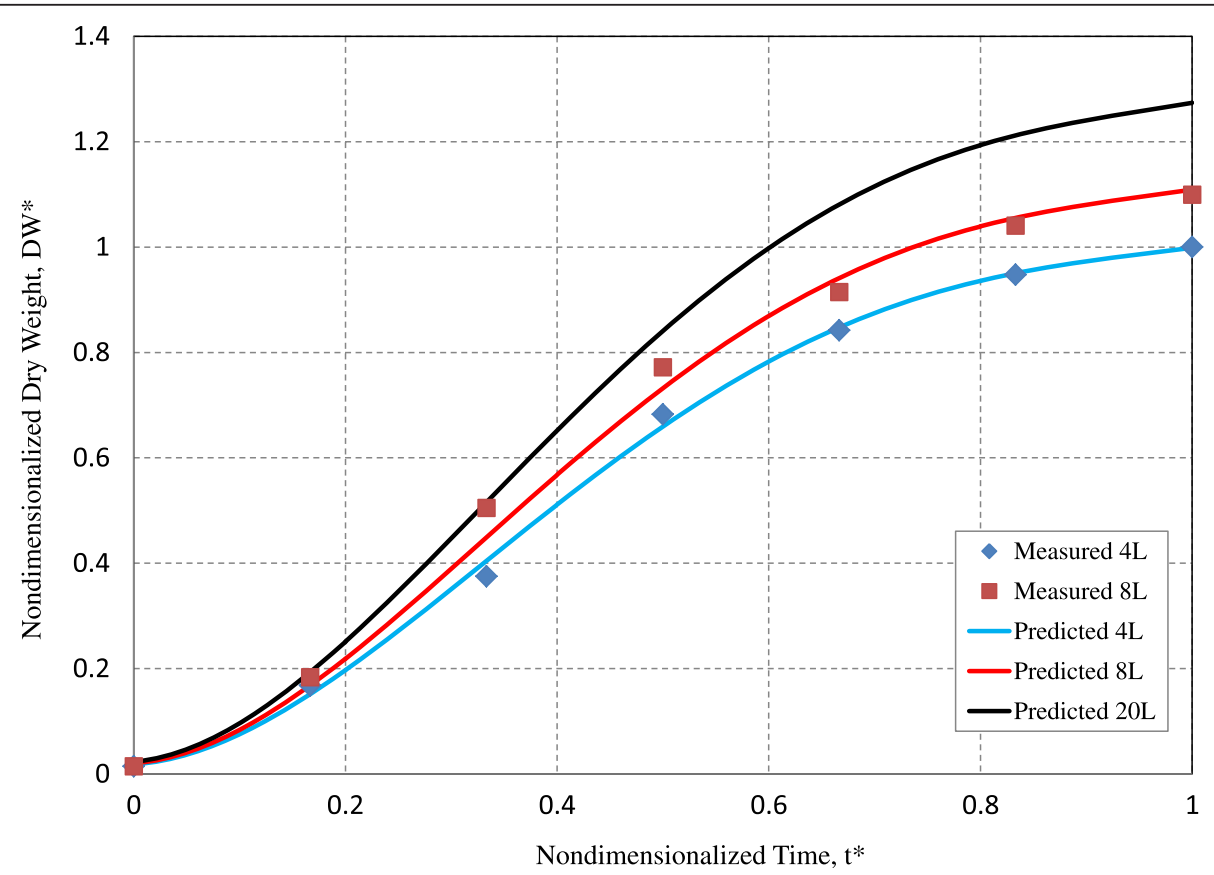

Figure 5 Nonlinear modeling for nondimensionalized dry weight from $4 \mathrm{~L}$ to $20 \mathrm{~L}$. 
nondimensional $\mathrm{DW}^{*}$ can be converted into dimensional DW in $\mathrm{g} / \mathrm{L}$ by multiplying by the maximum DW experimentally obtained from the baseline test which in this case is $4 \mathrm{~L}$. The nonlinear modeling agrees with measured data both qualitatively and quantitatively, where the modeling has enhanced the $\mathrm{R}^{2}$ value up to $95 \%$ compared to the linear model value $87.4 \%$ [16]. This suggests that the nonlinear regression approach enhances accuracy of modeling which provides key scale-up informations of $S$. bacillaris strain siva2011 biomass for $R R R$ - $\alpha$-tocopherol production. In this study, the significance of this empirical approach provides insights into the application of a nonlinear regression model that increases the $R^{2}$ value and enhances the quantitative predication of $S$. bacillaris strain siva2011 scaled-up in a $20 \mathrm{~L}$ bioreactor. This allows for the systematic understanding and design of a multi-variable nonlinear regression experiment for significant biomass production.

\section{Conclusions}

Photosynthetic microalgae are rich in $R R R$ - $\alpha$-tocopherol and a potential source for this natural antioxidant which is an essential human micronutrient. A significant advantage of this natural source is the maintenance of the specific bioactive form needed for nutrition and the elimination of possible issues with potential unknown or unexpected toxicities from the synthetic conformations. S. bacillaris strain siva2011 has a unique vitamin E biosynthetic mechanism capable of sustaining high levels of production, including inducible enhanced production which could provide a possible production platform of $R R R-\alpha$-tocopherol for pharmaceutical industries. A nonlinear mathematical model was developed to model scale-up to production in $20 \mathrm{~L}$ reactors using an approach with a higher accuracy based on the dual variables tested in $4 \mathrm{~L}$ and $8 \mathrm{~L}$ reactors. The $\mathrm{R}^{2}$ value from this study demonstrates that this nonlinear approach significantly improves estimation of $S$. bacillaris strain siva2011 biomass production in the bioreactor than does the linear model. Additional studies with progressively larger reactors (and models) will be needed to bridge the gap between laboratory and industrial scale. Nevertheless, this data provides enhanced bioprocess engineering information in the progression towards large-scale pharmaceutical $R R R$ - $\alpha$-tocopherol production from S. bacillaris strain siva2011 biomass.

\section{Methods}

\section{Bioreactor culture, elicitation and analytics}

S. bacillaris strain siva2011 cells were cultured in a balloon type bioreactor ( $4 \mathrm{~L}$ and $8 \mathrm{~L}$ ). The bioreactor experimental design and the biomass harvesting were performed as described by Sivakumar et al. [16]. For elicitation, three concentrations of filter sterilized MeJa 50, 100, and $200 \mu \mathrm{L} / \mathrm{L}$ were added to the $S$. bacillaris strain siva2011 culture on the $3^{\text {rd }}$ day. MeJa dissolved in ethanol and MeJa not dissolved in ethanol were tested, and both had a similar effect. Elicitations were carried out for 24, 48, or 72 hrs. Algal cells were harvested and freeze-dried according to the Sivakumar et al. [16] method. One gram of MeJa elicited and unelicited freeze-dried algal cells were used for analysis of $R R R-\alpha-$ tocopherol. $R R R$ - $\alpha$-tocopherol was processed according to the Sivakumar et al. [4] method. The reversed phasehigh performance liquid chromatography chromatogram was acquired using a Dionex 3000 (LPG3400A) system (Thermo Scientific, Sunnyvale, CA) equipped with a thermostatted UltiMate 3000 autosampler and a Dionex RF 2000 fluorescence detector. The system was monitored by the software's chromeleon (6.80) for instrument control and data acquisition, data reprocessing, and solute quantification, respectively. An Agilent Zorbax Eclipse XDB-C8 $(250 \mathrm{~mm} \times 4.6 \mathrm{~mm}$, mean particle size $5 \mu \mathrm{m})$ column or C18 $(250 \mathrm{~mm} \times 4.6 \mathrm{~mm}$, mean particle size $5 \mu \mathrm{m}$ ) was used to separate $R R R-\alpha$ tocopherol. The mobile phase consists of a linear gradient of $90 \%$ methanol in water. The flow rate was $1 \mathrm{ml} /$ min. The total acquisition time was $35 \mathrm{~min}$. The wavelength was set at $290 \mathrm{~nm}$ for excitation and $330 \mathrm{~nm}$ for emission. The authenticated $R R R$ - $\alpha$-tocopherol fluorescence spectra and retention time was used for HPLC confirmation of $R R R$ - $\alpha$-tocopherol in the samples. The $R R R$ - $\alpha$-tocopherol liquid chromatography mass spectrometry spectrum confirmation was acquired using a Shimadzu 8050 mass spectrometer.

\section{Nonlinear regression}

For a stepwise approach, the first phase of the nonlinear regression method begins with the simplification from five variables to two variables, time $(t)$ and reactor volume $(\mathrm{V})$, as shown in equation 1 . The regression will be extended to multivariables when the two variables method is validated.

$$
D W=f\left(y_{\mathrm{CO} 2}, E C, \mathrm{OrP}, p H, t, V\right) \approx f(t, V)
$$

The associated variables DW, $\mathrm{t}$, and $\mathrm{V}$ in equation 1 are nondimensionalized into $\mathrm{DW}^{*}, \mathrm{t}^{*}$, and $\mathrm{V}^{* *}$ as shown in equation (2) to (4).

$$
\begin{aligned}
& D W^{*}=\frac{D W}{D W_{\text {max }}} \\
& t^{*}=\frac{t}{t_{\max }} \\
& V^{*}=\frac{V[L]}{4 L}
\end{aligned}
$$

Where $\mathrm{DW}_{\max }$ is the maximum $\mathrm{DW}[\mathrm{g} / \mathrm{L}]$ produced from $4 \mathrm{~L}$ test under $0.02 \% \mathrm{CO}_{2}$ fraction, and $\mathrm{t}_{\max }$ is the 
maximum time to reach the maximum DW. In this study, $\mathrm{DW}_{\max }$ and $\mathrm{t}_{\max }$ were $3.45 \mathrm{~g} / \mathrm{L}$ and 6 days, respectively. The volume was standardized by the baseline: $4 \mathrm{~L}$ in this modeling.

A nonlinear model was assumed by combining $4^{\text {th }}$ order polynomials and the power form of the equation as shown in equation (5) in consideration of measured data of $4 \mathrm{~L}$ and $8 \mathrm{~L}$.

$$
D W^{*}=\left(a t^{* 4}+b t^{* 3}+c t^{* 2}+d t^{* 2}+e t+f\right) V^{* g}
$$

All associated constants a, b, c, and d in equation (5) were determined from a nonlinear regression method as shown in equation (6).

$$
\begin{aligned}
D W^{*}= & \left(2.686 t^{* 4}-6.942 t^{* 3}+5.109 t^{* 2}\right. \\
& +0.127 t+0.0184) V^{* 0.151}
\end{aligned}
$$

Equation (6) allows the predicting of DW in reactor volume $20 \mathrm{~L}$ at $0.02 \% \mathrm{CO}_{2}$ fraction from the correlation obtained from $4 \mathrm{~L}$ and $8 \mathrm{~L}$ measured data.

\section{Statistical analysis}

Bioreactor culture, elicitations, and analytical experiments were repeated at least three times, each with three replications. Results were expressed as the mean with standard errors. Stepwise dual nonlinear regressions were used to investigate the relationship between $4 \mathrm{~L}$ and $8 \mathrm{~L}$ in order to predict $20 \mathrm{~L}$ bioreactor scale-up.

\section{Abbreviations \\ C: Carbon; $\mathrm{CO}_{2}$ : Carbon dioxide; DW: Dry weight; G: Gram; hrs: Hours; a: Trimethyl; $\beta, y$ : Dimethyl; $\delta$ : Monomethyl; a-TTP: a-Tocopherol transfer protein; MeJa: Methyl jasmonate; Mg: Milligram; $\mu \mathrm{L}$ : Microliter; L: Liter; \%: Percentage; ROS: Reactive oxygen species.}

\section{Competing interests}

The authors declare that they have no competing interests.

\section{Authors' contributions}

GS, JL, and KJ made substantial contributions to the experimental design, analysis, and/or interpretation of data. Specifically, GS led and designed the experiments and performed the bioprocessing and HPLC studies. JL performed the mass spectrometry experiments for compound confirmation. $\mathrm{KJ}$ performed the mathematical modeling to validate the variables. GS wrote the manuscript which was reviewed and approved by all authors. All authors read and approved the final manuscript.

\section{Acknowledgments}

This research was funded by the Arkansas Biosciences Institute (grants 262178 and 200109). The technical assistance of Kelsea Brewer and Dez Jones (Arkansas State University, Jonesboro, AR, USA), and Dr. Jennifer Gidden (University of Arkansas, Fayetteville, AR, USA) was appreciated.

\section{Author details}

${ }^{1}$ Arkansas Biosciences Institute and College of Agriculture and Technology, Arkansas State University, PO Box 639, Jonesboro, AR 72401, USA. ${ }^{2}$ College of Engineering, Arkansas State University, Jonesboro, AR 72401, USA. ${ }^{3}$ Arkansas Statewide Mass Spectrometry Facility, University of Arkansas, Fayetteville, AR 72701, USA
Received: 11 April 2014 Accepted: 30 May 2014

Published: 3 June 2014

\section{References}

1. Dasilva EJ, Jensen A: Content of a-tocopherol in some blue-green algae. Biochim Biophys Acta 1971, 239:345-347.

2. Sivakumar G, Bacchetta L: Determination of natural vitamin E from Italian hazelnut leaves. Chem Nat Compd 2005, 6:654-656.

3. Maeda H, DellaPenna D: Tocopherol functions in photosynthetic organisms. Curr Opin Plant Biol 2007, 10:260-265.

4. Sivakumar G, Bacchetta L, Gatti R, Zappa G: HPLC screening of natural vitamin E from Mediterranean plant biofactories - a basic tool for pilot-scale bioreactors production of a-tocopherol. J Plant Physiol 2005, 162:1280-1283.

5. Niki E: Role of vitamin $\mathrm{E}$ as lipid-soluble peroxyl radical scavenger: in vitro and in vivo evidence. Free Radic Biol Med 2013, 66:3-12.

6. Bermudez Y, Ahmadi S, Lowell NE, Kruk PA: Vitamin E suppresses telomerase activity in ovarian cancer cells. Cancer Detect Prev 2007, 31:119-128.

7. Molano A, Meydani SK: Vitamin E, signalosomes and gene expression in T cells. Mol Aspects Med 2012, 33:55-62.

8. Pekmezci D: Vitamin E and immunity. Vitam Horm 2011, 86:179-215.

9. Yang CS, Li G, Yang Z, Guan F, Chen A, Ju J: Cancer prevention by tocopherols and tea polyphenols. Cancer Lett 2013, 334:79-85.

10. Kamimura W, Doi W, Takemoto K, Ishihara K, Wang DH, Sugiyama H, Oda S, Masuoka N: Effect of vitamin E on alloxan-induced mouse diabetes. Clin Biochem 2013, 46:795-798.

11. Röhrle FT, Moloney AP, Black A, Osorio MT, Sweeney T, Schmidt O, Monahan FJ: a-Tocopherol stereoisomers in beef as an indicator of vitamin E supplementation in cattle diets. Food Chem 2011, 124:935-940.

12. Ajjawi I, Shintani D: Engineered plants with elevated vitamin E: a nutraceutical success story. Trends Biotechnol 2004, 22:104-107.

13. Sivakumar G, Yu KW, Paek KY: Production of biomass and ginsenosides from adventitious roots of Panax ginseng in bioreactor cultures. Eng Life Sci 2005, 5:333-342.

14. Sivakumar G, Medina-Bolivar F, Lay JO, Dolan MC, Condori J, Grubbs SK, Wright SM, Baque MA, Lee EJ, Paek KY: Bioprocess and bioreactor: next generation technology for production of potential plant-based antidiabetic and antioxidant molecules. Curr Med Chem 2011, 18:79-90.

15. Sivakumar G: Bioreactor technology: a novel industrial tool for high-tech production of bioactive molecules and biopharmaceuticals from plant roots. Biotechnol J 2006, 1:1419-1427.

16. Sivakumar G, Jeong K, Lay JO: Bioprocessing of Stichococcus bacillaris strain siva2011. Biotechnol Biofuels 2014, 7:62.

17. Zingg ZM: Vitamin E: an overview of major research directions. Mol Aspects Med 2007, 28:400-422.

18. Munne-Bosch S: a-Tocopherol: a multifaceted molecule in plants. Vitam Horm 2007, 76:375-392

19. Liu K, Luo HL, Yue DB, Ge SY, Yuan F, Yan LY, Jia HN: Molecular cloning and characterization of the sheep a-TTP gene and its expression in response to different vitamin E status. Gene 2011, 494:225-230.

20. Jensen SK, Lauridsen C: Alpha-tocopherol stereoisomers. Vitam Horm 2007, 76:281-308.

21. Traber MG, Ramakrishnan R, Kayden HJ: Human plasma vitamin E kinetics demonstrate rapid recycling of plasma RRR-a-tocopherol. Proc Natl Acad Sci U S A 1994, 91:10005-10008.

22. Traber MG, Atkinson J: Vitamin $\mathrm{E}$, antioxidant and nothing more. Free Radic Biol Med 2007, 43:4-15.

23. Nowicka B, Kruk J: Plastoquinol is more active than a-tocopherol in singlet oxygen scavenging during high light stress of Chlamydomonas reinhardtii. Biochim Biophys Acta 1817, 2012:389-394.

24. Sarah C, Hunter SC, Cahoon EB: Enhancing vitamin E in oilseeds: unraveling tocopherol and tocotrienol biosynthesis. Lipids 2007, 42:97-108.

25. Porfirova S, Bergmuller E, Tropf S, Lemke R, Dormann P: Isolation of an Arabidopsis mutant lacking vitamin $\mathrm{E}$ and identification of a cyclase essential for all tocopherol biosynthesis. Proc Natl Acad Sci U S A 2002, 99:12495-12500.

26. Tsegaye $Y$, Shintani DK, DellaPenna D: Overexpression of the enzyme p-hydroxyphenylpyruvate dioxygenase in Arabidopsis and its relation to tocopherol biosynthesis. Plant Physiol Biochem 2002, 40:913-920. 
27. DellaPenna D: Progress in the dissection and manipulation of vitamin $\mathrm{E}$ synthesis. Trends Plant Sci 2005, 10:574-579.

28. Cahoon EB, Hall SE, Ripp KG, Ganzke TS, Hitz WD, Coughlan SJ: Metabolic redesign of vitamin $\mathrm{E}$ biosynthesis in plants for tocotrienol production and increased antioxidant content. Nat Biotechnol 2003, 21:1082-1087.

29. Sattler SE, Cahoon EB, Coughlan SJ, DellaPenna D: Characterization of tocopherol cyclases from higher plants and cyanobacteria. Evolutionary implications for tocopherol synthesis and function. Plant Physiol 2003, 132:2184-2195.

30. Backasch N, Schulz-Friedrich R, Appel J: Influences on tocopherol biosynthesis in the cyanobacterium Synechocystis sp. PCC 6803. J Plant Physiol 2005, 162:758-766.

31. Antognoni F, Faudale M, Poli F, Biondi S: Methyl jasmonate differentially affects tocopherol content and tyrosine amino transferase activity in cultured cells of Amaranthus caudatus and Chenopodium quinoa. Plant Biol (Stuttg) 2009, 11:161-169.

32. Caretto S, Nisi R, Paradiso A, De Gara L: Tocopherol production in plant cell cultures. Mol Nutr Food Res 2010, 54:726-730.

33. Kusmic C, Barsacchi R, Barsanti L, Gualtieri P, Passarelli V: Euglena gracilis as source of the antioxidant vitamin E. Effects of culture conditions in the wild strain and in the natural mutant WZSL. J Appl Psychol 1999, 10:555-559.

34. Carballo-Cardenas EC, Tuan PM, Janssen M, Wijff RH: Vitamin E (a-tocopherol) production by the marine microalgae Dunaliella tertiolecta and Tetraselmis suecica in batch cultivation. Biomol Eng 2003, 20:139-147.

35. Kruk J, Szymanska R, Krupinska K: Tocopherol quinone content of green algae and higher plants revised by a new high-sensitive fluorescence detection method using HPLC-Effects of high light stress and senescence. J Plant Physiol 2008, 165:1238-1247.

36. Mendiola JA, Garcıa-Martınez D, Ruperez FJ, Martın-Alvarez PJ, Reglero G, Cifuentes A, Barbas C, Ibanez E, Senorans FJ: Enrichment of vitamin E from Spirulina platensis microalga by SFE. J Supercrit Fluids 2008, 43:484-489.

37. Turner JG, Ellis C, Devoto A: The jasmonate signal pathway. Plant Cell 2002, 14:153-164

38. Cheong JJ, Choi YD: Methyl jasmonate as a vital substance in plants. Trends Genet 2003, 19:409-413.

39. Sandorf I, Holländer-Czytko H: Jasmonate is involved in the induction of tyrosine aminotransferase and tocopherol biosynthesis in Arabidopsis thaliana. Planta 2002, 216:173-179.

40. Seo HS, Song JT, Cheong JJ, Lee YH, Lee YW, Hwang I, Lee JS, Choi YD: Jasmonic acid carboxyl methyltransferase: a key enzyme for jasmonate-regulated plant responses. Proc Natl Acad Sci U S A 2001, 98:4788-4793.

41. Zhang ZP, Baldwin IT: Transport of [2-14C]jasmonic acid from leaves to roots mimics wound-induced changes in endogenous jasmonic acid pools in Nicotiana sylvestris. Planta 1997, 203:436-441.

42. Tamogami S, Noge K, Abe M, Agrawal GK, Rakwal R: Methyl jasmonate is transported to distal leaves via vascular process metabolizing itself into $\mathrm{JA}$-lle and triggering VOCs emission as defensive metabolites. Plant Signal Behav 2012, 7:1-4.

43. Zhang L, Xing D: Methyl jasmonate induces production of reactive oxygen species and alterations in mitochondrial dynamics that precede photosynthetic dysfunction and subsequent cell death. Plant Cell Physiol 2008, 49:1092-1111.

44. Wierstra I, Kloppstech K: Differential effects of methyl jasmonate on the expression of the early light-inducible proteins and other light-regulated genes in barley. Plant Physiol 2000, 124:833-844.

45. Bates $\mathrm{D}$, Watts $\mathrm{D}$ : Nonlinear regression analysis and its applications. NY, USA: Wiley-Interscience; 2007.

46. Liu S: Bioprocess engineering: kinetics, biosystems, sustainability, and reactor design. Oxford, UK: Elsevier; 2012.

47. Hassan SS, Farhan M, Mangayil R, Huttunen H, Aho T: Bioprocess data mining using regularized regression and random forests. BMC Syst Biol 2013, 7:S5.

48. Archontoulis SV, Miguez FE: Non-linear regression models and applications in agricultural research. Agronomy J 2013, 105:1-13.

doi:10.1186/1475-2859-13-79

Cite this article as: Sivakumar et al:: Biomass and RRR-a-tocopherol production in Stichococcus bacillaris strain siva2011 in a balloon bioreactor. Microbial Cell Factories 2014 13:79.

\section{Submit your next manuscript to BioMed Central and take full advantage of:}

- Convenient online submission

- Thorough peer review

- No space constraints or color figure charges

- Immediate publication on acceptance

- Inclusion in PubMed, CAS, Scopus and Google Scholar

- Research which is freely available for redistribution 